\title{
Dynamic Model and Motion Control of a Robotic Manipulator
}

\author{
Jinho Kim, Kevin Chang, Brian Schwarz \\ Department of Mechanical Engineering, University of Maryland, Baltimore County (UMBC), 1000 Hilltop Circle, \\ Baltimore, Maryland, USA, 21250 \\ Andrew S. Lee, S. Andrew Gadsden \\ School of Engineering, University of Guelph, \\ Guelph, Ontario, Canada, N1G2W1 \\ Mohammad Al-Shabi \\ Department of Mechanical Engineering, University of Sharjah, University City Rd, Sharjah, United Arab Emirates \\ E-mail:umbcjhkim@umbc.edu,alee32@uoguelph.ca,kevicha1@umbc.edu,schwarz4@umbc.edu, \\ malshabi@sharjah.ac.ae, and gadsden@uoguelph.ca
}

\begin{abstract}
This paper presents the dynamic modeling and motion control of a three-link rotary robotic manipulator, also known as an RRR robot. The Kinect motion capture system by Microsoft is used in conjunction with the manipulator. A camera is used to capture the motion of a user's arm and tracks certain angles made by parts of the arm. We consider a pinhole camera model to generate reference angles in our simulations. These desired angles are fed into the controller and are used by the RRR robot in an effort to copy the movement of the user. A proportionalderivative (PD) controller is developed and applied to the manipulator for improved trajectory tracking. The RRR robot is dynamically modeled and the results of the proposed control strategy demonstrate good trajectory following. Researchers can implement this system as a relatively inexpensive starting point for their activities.
\end{abstract}

Keywords: Three-link robotic manipulator, RRR robot manipulator, Motion capture, PD controller.

\section{Introduction}

Robotic manipulators have been developed and utilized in a variety of sectors, including: industry, medical, and military. In recent years, due to their potential to improve precision and enhance robustness, robotic manipulators have been applied to not only military missions but also medical devices. There are many research topics for medical devices with robotic manipulators such as human-robot collaboration, motion planning, and simultaneous control [1-4]. In the military field, we can easily perform robotic manipulator applications as well. For instance, autonomous ground vehicles with robotic manipulators are used in bomb disarming, search and rescue, and reconnaissance. To conduct surgery or translate human movement with a robotic manipulator on military missions remotely, it is important to control the robotic manipulator in sync with the movement of the operator [5-13].

In this brief paper, we consider controlling a system which consists of a camera and robotic manipulator. The pinhole camera model extracts reference angles from simulations. The desired angles are fed into the controller and are utilized by the robotic manipulator in an effort to mimic the movement of the user. A proportional-derivative (PD) controller is designed and 
applied to the robotic manipulator for trajectory tracking. The paper is organized as follows. In Section 2 , the pinhole camera model is introduced and the threelink robotic manipulator model is derived using the classical Euler-Lagrange equation. Based on these models, a PD controller is designed and shown in Section 3. The simulation results are presented and discussed in Section 4. Finally, the paper concludes and future work is described.

\section{Pinhole Camera Model}

The pinhole camera model is used to obtain reference angles. The camera captures the motion of the user's arm. The shoulder, elbow, and wrist angles are extracted through image processing. The pinhole camera model is shown in Fig. 1.

Note that we define $\mathbb{I}$ as the inertial coordinate

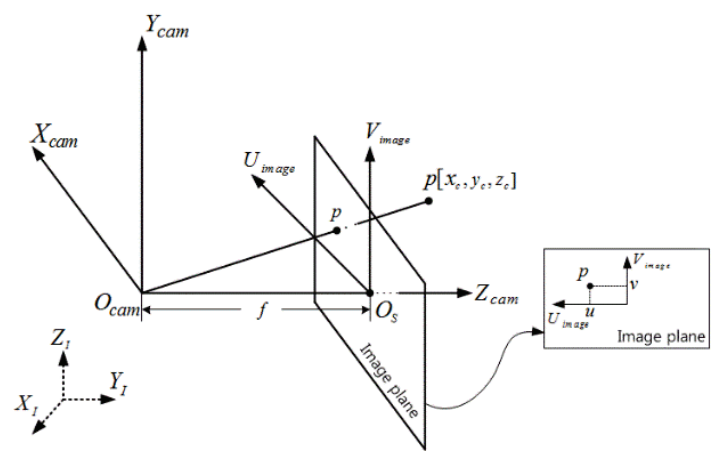

Figure 1. Geometry and coordinate frames for the pinhole camera model.

frame, $\mathbb{C}$ as the camera frame, and $\mathbb{S}$ as the image frame. Let $\left[X_{I}, Y_{I}, Z_{I}\right]^{T}$ be the axes of the inertial coordinate frame II, and $\left[X_{\text {cam }}, Y_{\text {cam }}, Z_{\text {cam }}\right]^{T}$ be the position vector in the camera frame $\mathbb{C}$. Let $\left[U_{\text {image }}, V_{\text {image }}\right]^{T}$ be the coordinate axes of the image frame $\mathbb{S}$ and $O_{S}$ represents the principal point where the $z$-axis of the camera coordinate system intersects the image plane. The focal length of the camera, $f$, is the distance between $O_{c a m}$ and $O_{S}$. Finally, the mapping from a point $P=$ $\left[x_{c}, y_{c}, z_{c}\right]^{T} \in \mathbb{C}$ onto the image plane can be written as Eq.(1).

$$
p=\left[\begin{array}{l}
u \\
v
\end{array}\right]=\frac{f}{z_{c}}\left[\begin{array}{l}
x_{c} \\
y_{c}
\end{array}\right]
$$

The user's arm is projected onto the image plane $\mathbb{S}$ through Eq. (1). The three angles are calculated by extracting three points from the image plane using simple geometry. Let us define two points in $\mathbb{S}$ as $p_{1}=\left(u_{1}, v_{1}\right)$ and $p_{2}=\left(u_{2}, v_{2}\right)$. The angle can be calculated as follows:

$$
\theta=\tan ^{-1}\left(\frac{v_{2}-v_{1}}{u_{2}-u_{1}}\right)
$$

\section{Dynamics and PD Controller for the RRR Robotic Manipulator}

In this section, we derive the dynamics of a three-link robotic manipulator and design a PD controller for it. An overview of the system is depicted in Fig. 2.

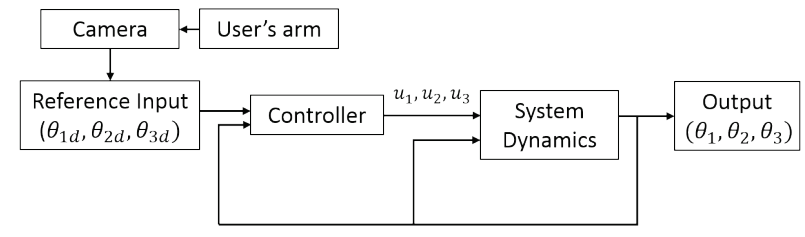

Figure 2. Overview of the system.

\subsection{Dynamics of Robotic Manipulator}

The configuration of the three-link robotic manipulator is shown in Fig. 3. Note that $\theta_{i}(i=1,2,3)$ denotes the angles between each link, $L_{i}$ is the length of the link, $l_{i}$ is the distance from the centroid to corresponding axis, $m_{i}$ is the mass, and $J_{i}$ is the moment of inertia. Let $\tau_{i}$ denotes the joint torque and its counterclockwise rotation is assumed to be positive. The Euler-Lagrange equation is applied as shown in Eqs. (3-4) where $\mathcal{L}$ is the Lagrangian with kinetic energy $\mathcal{K}$ and potential energy $\mathcal{P}$ of the system [10].

$$
\begin{gathered}
\frac{d}{d t} \frac{\partial \mathcal{L}}{\partial \dot{\theta}_{l}}-\frac{\partial \mathcal{L}}{\partial \theta_{i}}=\tau_{i} \\
\mathcal{L}=\mathcal{K}_{1}+\mathcal{K}_{2}+\mathcal{K}_{3}-\mathcal{P}_{1}-\mathcal{P}_{2}-\mathcal{P}_{3}
\end{gathered}
$$

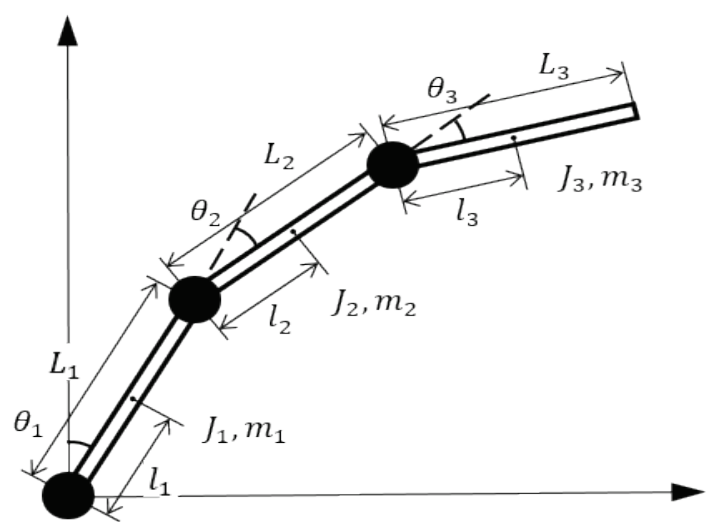

Figure 3. Configuration of the three-link robotic manipulator. 
In this system, $\mathcal{K}$ and $\mathcal{P}$ of each link can be written as per Eqs. (5-6) where $\left\|v_{i}\right\|$ represents the magnitude of the velocity, $l_{i, y}$ is the position of the centroid that is dependent on joint coordinates.

$$
\begin{gathered}
\mathcal{K}_{i}=\frac{1}{2} m_{i}\left\|v_{i}\right\|^{2}+\frac{1}{2} I_{i}\left(\sum_{i} \dot{\theta}_{i}\right)^{2} \\
P_{i}=m_{i} g l_{i, y}
\end{gathered}
$$

From these equations, we can obtain the final dynamics of the system in a simple form as follows:

$$
u=M(\theta) \ddot{\theta}+C(\theta, \dot{\theta})+g(\theta)
$$

where $M(\theta)$ is the inertia matrix, $C(\theta, \dot{\theta})$ represents the matrix of coriolis and centrifugal elements, $g(\theta)$ is the matrix of gravity elements, and, $u$ is control input. The matrices and their parameters can be written as follows:

$$
\begin{gathered}
M(\theta)=\left[\begin{array}{lll}
a_{11} & a_{12} & a_{13} \\
a_{12} & a_{22} & a_{23} \\
a_{13} & a_{23} & a_{33}
\end{array}\right], \\
C(\theta, \dot{\theta})=\left[\begin{array}{l}
b_{1} \\
b_{2} \\
b_{3}
\end{array}\right], \\
g(\theta)=\left[\begin{array}{l}
g_{1} \\
g_{2} \\
g_{3}
\end{array}\right],
\end{gathered}
$$

where,

$$
\begin{aligned}
& a_{11}=m_{1} a_{c 1}^{2}+m_{2}\left(a_{1}^{2}+a_{c 2}^{2}+2 a_{1} a_{c 2} c_{2}\right)+m_{3}\left(a_{1}^{2}+a_{2}^{2}+\right. \\
& \left.a_{c 3}^{2}+2 a_{1} a_{2} c_{2}+2 a_{1} a_{c 3} c_{23}+2 a_{2} a_{c 3} c_{3}\right)+I_{1}+I_{2}+I_{3} \\
& a_{12}=m_{2}\left(a_{c 2}^{2}+a_{1} a_{c 2} c_{2}\right)+m_{3}\left(a_{2}^{2}+a_{c 3}^{2}+a_{1} a_{2} c_{2}+\right. \\
& \left.a_{1} a_{c 3} c_{23}+2 a_{2} a_{c 3} c_{3}\right)+I_{2}+I_{3} \\
& a_{13}=m_{3}\left(a_{c 3}^{2}+a_{1} a_{c 3} c_{23}+a_{2} a_{c 3} c_{3}\right)+I_{3} \\
& a_{22}=m_{2} a_{c 2}^{2}+m_{3}\left(a_{2}^{2}+a_{c 3}^{2}+2 a_{2} a_{c 3} c_{3}\right)+I_{2}+I_{3} \\
& a_{23}=m_{3}\left(a_{c 3}^{2}+a_{2} a_{c 3} c_{3}\right)+I_{3} \\
& a_{33}=m_{3} a_{c 3}^{2}+I_{3} \\
& b_{1}=-m_{2} a_{1} a_{c 2}\left(2 \dot{\theta}_{1}+\dot{\theta}_{2}\right) s_{2} \dot{\theta}_{2}-m_{3}\left[a_{1} a_{2}\left(2 \dot{\theta}_{1}+\dot{\theta}_{2}\right) s_{2}+\right. \\
& \left.a_{1} a_{c 3}\left(2 \dot{\theta}_{1}+\dot{\theta}_{2}+\dot{\theta}_{3}\right) s_{23}+a_{2} a_{c 3}\left(2 \dot{\theta}_{1}+2 \dot{\theta}_{2}+\dot{\theta}_{3}\right) s_{3}\right] \\
& b_{2}=-m_{2}\left[a_{1} a_{c 2}\left(\dot{\theta}_{1}^{2}+\dot{\theta}_{1} \dot{\theta}_{2}\right) s_{2}+a_{1} a_{c 2} \dot{\theta}_{1} s_{2} \dot{\theta}_{2}\right]- \\
& m_{3}\left[a_{1} a_{2}\left(\dot{\theta}_{1}^{2}+\dot{\theta}_{1} \dot{\theta}_{2}\right)+a_{1} a_{c 3}\left(\dot{\theta}_{1}^{2}+\dot{\theta}_{1} \dot{\theta}_{2}+\dot{\theta}_{1} \dot{\theta}_{3}\right) s_{23}+\right. \\
& a_{2} a_{c 3}\left(\dot{\theta}_{1}^{2}+2 \dot{\theta}_{1} \dot{\theta}_{2}+\dot{\theta}_{1} \dot{\theta}_{3}+\dot{\theta}_{2}^{2}+\dot{\theta}_{2} \dot{\theta}_{3}\right) s_{3}+a_{1} a_{2} \dot{\theta}_{1} s_{2} \dot{\theta}_{2}+ \\
& \left.a_{1} a_{c 3} \dot{\theta}_{1} \dot{\theta}_{2} s_{23}+a_{2} a_{c 3}\left(2 \dot{\theta}_{1}+2 \dot{\theta}_{2}+\dot{\theta}_{3}\right) s_{3}\right] \\
& b_{3}=-m_{3}\left[a_{1} a_{c 3}\left(\dot{\theta}_{1}^{2}+\dot{\theta}_{1} \dot{\theta}_{2}+\dot{\theta}_{1} \dot{\theta}_{3}\right) s_{23}+a_{2} a_{c 3}\left(\dot{\theta}_{1}^{2}+\right.\right. \\
& \left.2 \dot{\theta}_{1} \dot{\theta}_{2}+\dot{\theta}_{1} \dot{\theta}_{3}+\dot{\theta}_{2}^{2}+\dot{\theta}_{2} \dot{\theta}_{3}\right) s_{3}+a_{1} a_{c 3} \dot{\theta}_{1} \dot{\theta}_{3} s_{23}+ \\
& \left.a_{2} a_{c 3}\left(\dot{\theta}_{1}+\dot{\theta}_{2}\right) s_{3} \dot{\theta}_{3}\right] \\
& g_{1}=g\left[c_{1}\left(m_{1} a_{c 1}+m_{2} a_{1}+m_{3} a_{1}\right)+c_{12}\left(m_{2} a_{c 2}+m_{3} a_{2}\right)+\right. \\
& \left.c_{123}\left(m_{3} a_{c 3}\right)\right] \\
& g_{2}=g\left[\left(m_{2} a_{c 2}+m_{3} a_{2}\right) c_{12}+m_{3} a_{c 3} c_{123}\right] \\
& g_{3}=g\left(m_{3} a_{c 3} c_{123}\right) .
\end{aligned}
$$

\subsection{PD Controller}

In this subsection, the PD controller for the three-link manipulator system is presented.
In order to control the system, $\tau_{i}$ can be defined as a control input $u_{i}$ which controls $\theta_{i}$ with a reference input $\theta_{r}$. The PD controller of the system are given by:

$$
u_{i}=k_{p, i} e_{i}+k_{d, i} \dot{e}_{\imath}
$$

where $k_{p}$ and $k_{d}$ are proportional and derivative gains, respectively. The angle error, $e_{i}$, denotes $\theta_{r, i}-\theta_{i}$ and the derivative of the angle error, $\dot{e}_{l}$, denotes $\dot{\theta}_{r, i}-\dot{\theta}_{i}$.

\section{Simulation Results}

This section presents simulation results with initial parameters. The simulation used the initial settings which were defined as follows:

$$
\begin{gathered}
k_{p}=[37.5,24.2,16.4] \\
k_{d}=[1.34,0.87,1.04] \\
m_{i}=[1 \mathrm{~kg}, 1 \mathrm{~kg}, 1 \mathrm{~kg}] \\
L_{i}=[0.2 \mathrm{~m}, 0.2 \mathrm{~m}, 0.2 \mathrm{~m}], l_{i}=[0.1 \mathrm{~m}, 0.1 \mathrm{~m}, 0.1 \mathrm{~m}] \\
J_{i}=\left[0.5 \mathrm{~kg} \cdot \mathrm{m}^{2}, 0.5 \mathrm{~kg} \cdot \mathrm{m}^{2}, 0.5 \mathrm{~kg} \cdot \mathrm{m}^{2}\right] .
\end{gathered}
$$

We generated movements of arm and extracted the shoulder, elbow, and wrist angles as reference inputs $\theta_{r, 1}, \theta_{r, 2}, \theta_{r, 3}$. We set the reference inputs as follows:

$$
\begin{aligned}
& \theta_{r, 1}=3 \sin (0.1 \pi t) \\
& \theta_{r, 2}=4 \cos (0.1 \pi t) \\
& \theta_{r, 3}=9 \sin (0.15 \pi t)
\end{aligned}
$$

where $t$ is time from 0 to 25 seconds in this simulation. The history of each angle is shown in Fig. 4 where the black dashed lines are reference inputs $\theta_{r, i}$ and colored lines are current angles. As shown in Fig. 4, we can observe that the trajectories of the system angles follow the reference angels with satisfactory results. The initial overshoot in Fig. 4 shows acceptable performance of the PD controller for this system.
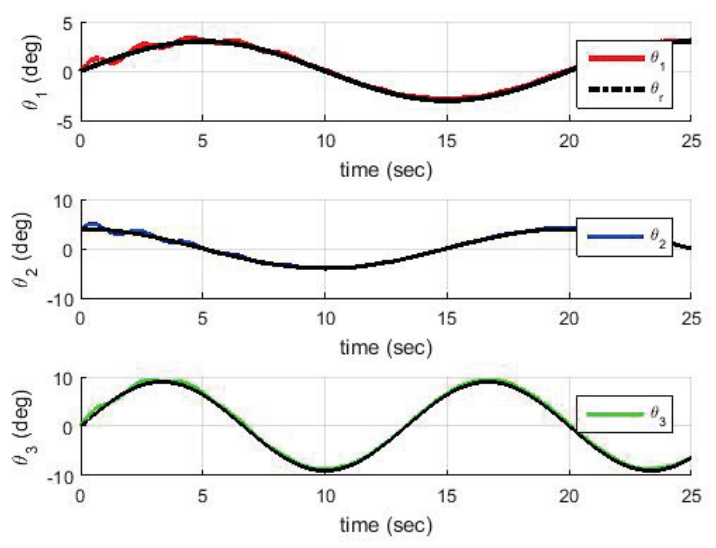

Figure 4. Results of PD controller for RRR robot. 


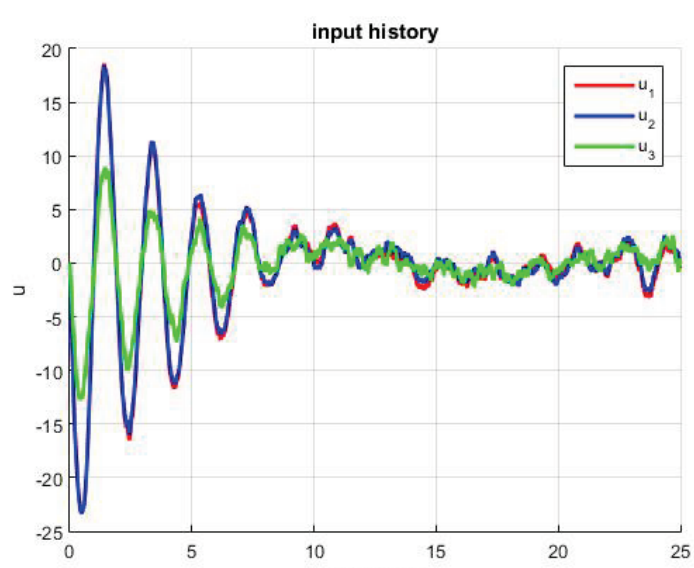

Figure 5. History of the three control inputs.

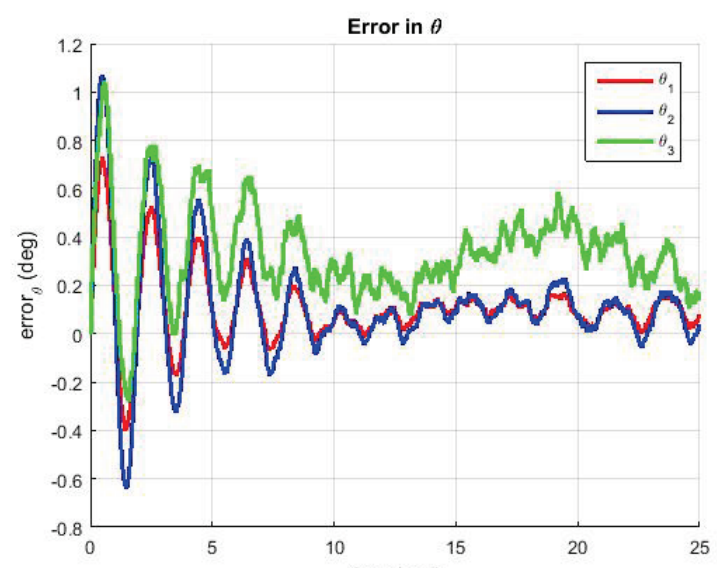

Figure 6. Error between angles and reference inputs.

The control signals and corresponding tracking errors are shown in Figs. 5 and 6, respectively. The magnitude errors are less than one degree which is acceptable for a robotic manipulator with linkage dimensions similar to a human arm.

\section{Conclusions}

This paper presents a three-link robotic manipulator combined with a camera for capturing the motion of the user. For successful control of the system, the pin hole camera model captures the arm motion of the user and extracts the shoulder, elbow, and wrist angles through image processing. The dynamics of the system are derived and the PD controller is applied to the angles of each link. The simulation results verify the efficacy of a PD controller and demonstrate satisfactory results for tracking arm movements.

\section{References}

1. S. Najarian, M. Fallahnezhad, and E. Afshari, Advances in medical robotic systems with specific applications in surgery-A review, Journal of medical engineering \& technology, 35(1) (2011) 19-33.

2. H. Jiang, J. P. Wachs, and B. S. Duerstock, Integrated vision-based robotic arm interface for operators with upper limb mobility impairments, Proc. 2013 IEEE International Conference on Rehabilitation Robotics (ICORR), (USA, Seattle, Jun. 2013), pp.1-6.

3. T. S. Lendvay, T. C. Brand, L. White, T. Kowalewski, S. Jonnadula, L. D. Mercer, D. Khorsand, J. Andros, B. Hannaford, and R. M. Satava, Virtual reality robotic surgery warm-up improves task performance in a dry laboratory environment: a prospective randomized controlled study, Journal of the American College of Surgeons,216(6) (2013): 1181-1192.

4. B. Hannaford, J. Rosen, D. W. Friedman, H. King, P. Roan, L. Cheng, D. Glozman, J. Ma, S. N. Kosari, and L. White. Raven-II: an open platform for surgical robotics research, IEEE Transactions on Biomedical Engineering, 60(4) (2013): 954-959.

5. X. Jian and L. Zushu, Dynamic model and motion control analys is of three-link gymnastic robot on horizontal bar, in Proc. The 2003 IEEE International Conference on Robotics and Intelligent Systems and Signal Processing, (China, Changsha, Oct. 2003).

6. I. David and G. Robles, PID control dynamics of a Robotic arm manipulator with two degrees of Freedom, Control de Procesos y Robotica (2012): 1-7.

7. B. Siciliano, S. Lorenzo, V. Luigi, and O. Giuseppe, Robotics: modelling, planning and control, (Springer Science \& Business Media, 2010).

8. M.W. Spong, Swing Up Control of the Acrobot, in Proc. The International Conference on Robotics and Automation, (USA, San Diego, Oct. 1994), pp.23562361.

9. M.W. Spong, The swing up control problem for the acrobot, IEEE Control System Magazine, 15(1) (1995): 49-55.

10. M. W. Spong, S. Hutchinson, and M. Vidyasagar, Robot modeling and control, (Wiley, New York, 2006).

11. S. A. Gadsden, Smooth Variable Structure Filtering: Theory and Applications, Ph.D. Thesis, McMaster, 2011.

12. J. Goodman, J. Kim, A. S. Lee, S. A. Gadsden, and M. Al-Shabi, Nonlinear Estimation Strategies Applied on an RRR Robotic Manipulator, The 2017 International Conference on Artificial Life and Robotics(ICAROB2017), Miyazaki, Japan, 2017.

13. J. Kim, A. S. Lee, K. Chang, B. Schwarz, S. A. Gadsden, and M. Al-Shabi, Dynamic Modeling and Motion Control of a Three-Link Robotic Manipulator, The 2017 International Conference on Artificial Life and Robotics(ICAROB2017), Miyazaki, Japan, 2017.

14. S. A. Gadsden and M. Al-Shabi, A Comparis on of Vibration Control Strategies for a Flexible-Link Robot Arm, 2015 IEEE International Symposium on Mechatronics and its Applications, Sharjah, UAE, 2015. 